\title{
Incidence and outcome of isolated distal deep vein thrombosis in kidney transplant recipients
}

\author{
Mi-Hyeong Kim¹, Kang-Woong Jun², Jeong-Kye Hwang ${ }^{1}$, Sun-Cheol Park ${ }^{3}$, Sang-Seop Yun ${ }^{3}$, In-Sung Moon ${ }^{4}$, \\ $\mathrm{Ji}-$ II Kim ${ }^{5}$ \\ 'Division of Vascular and Transplant Surgery, Department of Surgery, Eunpyeong St. Mary's Hospital, College of Medicine, The \\ Catholic University of Korea, Seoul, Korea \\ 2Division of Vascular and Transplant Surgery, Department of Surgery, Bucheon St. Mary's Hospital, College of Medicine, The \\ Catholic University of Korea, Bucheon, Korea \\ ${ }^{3}$ Division of Vascular and Transplant Surgery, Department of Surgery, Seoul St. Mary's Hospital, College of Medicine, The Catholic \\ University of Korea, Seoul, Korea \\ ${ }^{4}$ Division of Vascular and Transplant Surgery, Department of Surgery, Yeouido St. Mary's Hospital, College of Medicine, The \\ Catholic University of Korea, Seoul, Korea \\ ${ }^{5}$ Division of Vascular and Transplant Surgery, Department of Surgery, Uijeongbu St. Mary's Hospital, College of Medicine, The \\ Catholic University of Korea, Uijeongbu, Korea
}

\begin{abstract}
Purpose: Isolated distal deep vein thrombosis (IDDVT) is a localized lesion; nonetheless, the risk of proximal vein propagation makes it difficult to decide on the treatment protocol-that is, whether immediate anticoagulation after diagnosis or surveillance via serial imaging should be employed. This study aimed to investigate the appropriate treatment protocol for IDDVT in kidney transplant recipient (KTR).

Methods: Surveillance for venous thromboembolism (VTE) was performed using duplex ultrasonography (DUS) before transplant surgery and at 1 week, 2 weeks, 1 month, 3 months, 6 months, and 1 year after transplant surgery. Dual mechanical prophylaxis was administered to prevent VTE.

Results: In total, 60 out of 829 patients (7.2\%) developed VTE, with 49 cases (81.6\%) of IDDVT. Among IDDVT patients, 15 patients were treated using anticoagulation-first strategy, whereas the remaining 34 patients were treated using surveillance-first strategy. No patient in either group exhibited thrombus extension into the proximal vein or recurrence within 1 year from transplant surgery.

Conclusion: In Korean KTR, extension of IDDVT into the proximal vein could be prevented safely and effectively by serial DUS surveillance along with the maintenance of mechanical prophylaxis. However, the patients who have high thrombus burden or are difficult to get repeated DUS require the use of anticoagulation in early stages.

[Ann Surg Treat Res 2020;98(6):324-331]
\end{abstract}

Key Words: Anticoagulants, Kidney transplantation, Venous thrombosis

\section{INTRODUCTION}

Isolated distal deep vein thrombosis (IDDVT) refers deep vein thrombosis (DVT) localized to the infrapopliteal veins (peroneal, posterior tibial, anterior tibial, and muscular calf veins) without involvement of the proximal vein including the
Received October 7, 2019, Revised March 2, 2020, Accepted April 7, 2020

Corresponding Author: Ji-II Kim

Division of Vascular and Transplant Surgery, Department of Surgery, Uijeongbu St. Mary's Hospital, College of Medicine, The Catholic University of Korea, 271 Cheonbo-ro, Uijeongbu 11765, Korea

Tel: +82-31-820-3114, Fax: +82-31-847-2717

E-mail: cmckji@catholic.ac.kr

ORCID: https://orcid.org/0000-0001-5910-6916

\section{Copyright (c) 2020, the Korean Surgical Society}

(c) Annals of Surgical Treatment and Research is an Open Access Journal. All articles are distributed under the terms of the Creative Commons Attribution NonCommercial License (http://creativecommons.org/licenses/by-nc/4.0/) which permits unrestricted non-commercial use, distribution, and reproduction in any medium, provided the original work is properly cited. 
popliteal vein and above. Conflicting opinions on the clinical significance of this disease exist, with some evaluating IDDVT as a benign, localized, and self-limited disease with very low probability of propagating into the proximal vein $(<3 \%)$ in the absence of anticoagulation [1-3]. Nonetheless, others suggest the significance of IDDVT, which has been reported to extend into the proximal vein in $20 \%-30 \%$ of patients and to induce pulmonary thromboembolism in $5 \%-15 \%$ of patients in the absence of appropriate anticoagulation [4,5]. The American College of Chest Physicians guideline recommends that patients with severe symptoms and risk factors for proximal vein propagation should initially receive anticoagulant therapy, whereas those patients without severe symptoms should be monitored for propagation via serial imaging follow-up every 2 weeks [6].

In 2015, our group previously reported the characteristics of DVT that occurs after kidney transplantation (KT) [7]. Surveillance of $393 \mathrm{KT}$ recipients (KTR) via duplex ultrasonography (DUS) revealed that 18 out of 393 recipients had DVT, indicating a high incidence rate of $4.6 \%$. Of these DVT cases, $72 \%$ were in the form of IDDVT. Differentiating symptomatic DVT in KTR is difficult owing to the commonly observed leg swelling and increased D-dimer level, which mainly result from hypervolemia, hypoproteinemia, and steroid use. Furthermore, the risk of venous thromboembolism (VTE) occurrence in KTR increases compared with that in the general

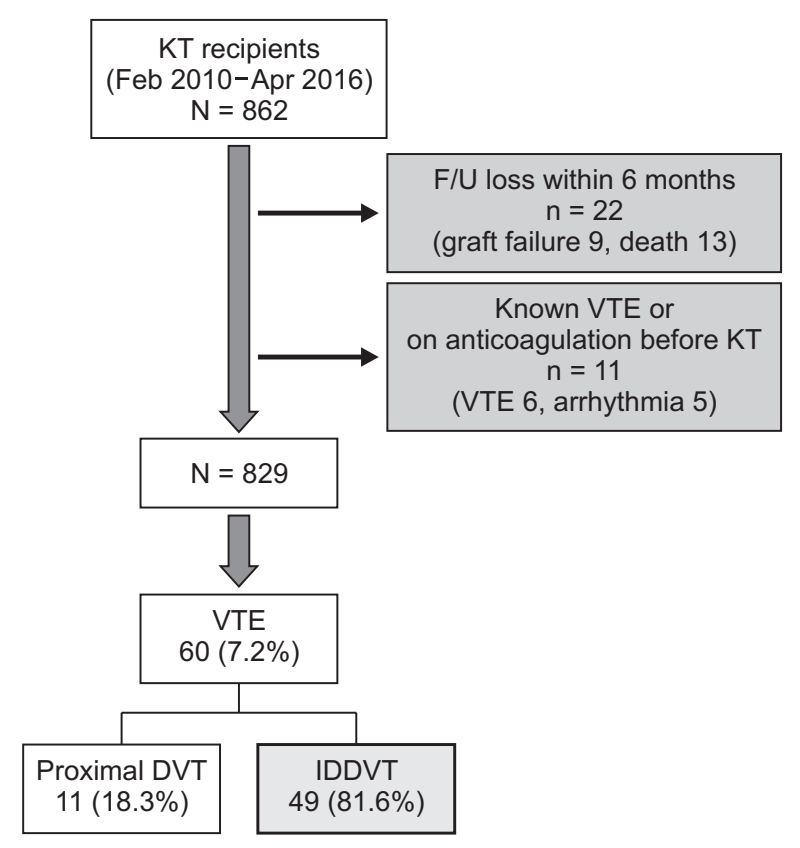

Fig. 1. Summary of patient selection. Total 829 kidney transplantation (KT) recipients were included and 49 patients were diagnosed isolated distal deep vein thrombosis (IDDVT). F/U, follow-up; VTE, venous thromboembolism; DVT, deep vein thrombosis. population because of the following reasons: hypercoagulability acquired under end-stage renal disease conditions prior to KT, manipulation of the iliac vein during surgery, immunosuppressant use after KT, and decreased mobility [813]. Nevertheless, no consensus on the therapeutic protocol for post-KT IDDVT exists. In addition, the use of anticoagulant therapy after KT is largely limited owing to multiple reasons, including bleeding tendency, insufficient renal function, drug interaction, and frequent invasive procedures (e.g., graft biopsy, urinary tract interventions). Therefore, it is difficult to decide on the therapeutic protocol for patients. Consequently, the present study aimed to investigate the appropriate treatment protocol for IDDVT in KTR and compare the outcomes of anticoagulation-first and surveillance-first strategies.

\section{METHODS}

\section{Patient selection and definition}

Medical records of a cohort of KTR who underwent surgery at a single institute between February 2010 and April 2016 were retrospectively reviewed. KTR diagnosed with IDDVT (both symptomatic and asymptomatic) by ultrasonography within 6 months of KT were included in this study. Conversely, those (1) who had DVT on preoperative ultrasonography or received anticoagulant therapy for various reasons; (2) who were diagnosed with calf vein thrombosis, along with proximal vein thrombosis and/or pulmonary thromboembolism; and (3) who were lost to follow-up within 6 months or refused to undergo serial follow-up examinations were excluded from the analysis (Fig. 1).

Changes in treatment protocol for IDDVT occurred in our center at different periods (Fig. 2). During period 1 (February 2010-March 2013), following diagnosis of IDDVT, patients

\begin{tabular}{|c|c|}
\hline $\begin{array}{c}\text { Period } 1 \\
\text { Anticoagulation first }\end{array}$ & $\begin{array}{c}\text { Period } 2 \\
\text { Surveillance first }\end{array}$ \\
\hline Feb 2010-Jun 2013 & Jul 2013-Apr 2016 \\
\hline \multicolumn{2}{|c|}{ Regardless of period... } \\
\hline $\begin{array}{l}\text { - Markedly symptomatic } \\
\text { - Extensive thrombus } \\
\text { : >5 cm in length } \\
>7 \mathrm{~mm} \text { in diameter } \\
\text { multiple veins } \\
\Rightarrow \text { Anticoagulation first }\end{array}$ & $\begin{array}{l}\text { - Thrombus confined to the } \\
\text { single muscular vein } \\
\text { - Contraindication of } \\
\text { anticoagulation } \\
\Rightarrow \text { Surveillance first }\end{array}$ \\
\hline
\end{tabular}

Fig. 2. The different strategies for isolated distal deep vein thrombosis (IDDVT) management. There were differences in IDDVT management strategy according to the time period. During period 1, the patients diagnosed with IDDVT immediately started anticoagulation treatment. During period 2 , the patients underwent surveillance examination every $1-2$ weeks to decide whether or not the patient should receive anticoagulation treatment. 
received anticoagulant therapy first. However, during period 2 (July 2013-April 2016), patients diagnosed with IDDVT did not receive immediate anticoagulant therapy but instead underwent follow-up DUS after 2 weeks; they received anticoagulant therapy if there was propagation. Patients with localized calf vein thrombosis or contraindications to anticoagulant therapy were treated using surveillance-first strategy irrespective of period. On the contrary, the following patients were treated using anticoagulation-first strategy irrespective of period: (1) patients with $\geq 3-\mathrm{cm}$ difference in diameter compared to the other leg or marked swelling compared to preoperative conditions and (2) patients with high thrombus burden (i.e., thrombus length $>5 \mathrm{~cm}$, thrombosed vein diameter $>7 \mathrm{~mm}$, involvement of multiple calf veins).

Disease progression was defined as thrombus extension into the proximal vein on follow-up DUS or additional thrombus observed in a different calf vein.

\section{Data collection and analysis}

The following general medical information and transplantand thrombosis-related data of patients were collected:

(1) General medical information: age, sex, height, weight, body mass index, medical history (cause of end-stage renal disease, diabetes, hypertension, previous VTE, or malignant tumors), preoperative anticoagulant use, hormone therapy, dialysis (modality and duration), erythropoietin-based treatment, and history of smoking

(2) Transplant-related data: history of organ transplantation, donor type (living or deceased donor organ transplant), immunological risk (ABO-incompatible or human leukocyte antigen [HLA]-incompatible), desensitization treatment, type of induction agent (antithymocyte globulin [ATG] or basiliximab), renal function immediately after KT, graft survival, and patient survival

(3) Thrombosis-related data: preoperative blood test (platelet count, hematocrit, prothrombin time, D-dimer, fibrinogen, protein $\mathrm{C}$, protein $\mathrm{S}$, antithrombin III, factor V Leiden mutation, prothrombin 20210A mutation, lupus anticoagulant, homocysteine, anticardiolipin antibody), type and duration of anticoagulant therapy, and complications (thrombus- and anticoagulation-related)

Using the above data collected, we performed a historical comparison of medical characteristics and clinical outcomes of patients during both periods 1 (anticoagulation first) and 2 (surveillance first). Data from both groups are expressed as mean \pm standard deviation (range) or percentage (\%). The endpoints of this study were the efficacy and safety of the 2 treatment strategies, and disease progression and thrombusand anticoagulation-related complications were compared between the 2 groups.

This study was performed with approval from the
Institutional Review Board of the Catholic University of Korea Seoul St. Mary's Hospital (KC19RESI0042). The requirement for acquisition of informed consent from subjects was waived owing to the retrospective nature of our study.

\section{VTE surveillance and prophylaxis protocol}

In our center, DUS is performed before transplant surgery and at 1 week, 2 weeks, 1 month, 3 months, 6 months, and 1 year after transplant surgery. During examination, the following aspects were evaluated: status of renal graft perfusion and patency of anastomosis; status of the ureter; status of perigraft space; and flow in and patency of the iliac, femoral, popliteal, and calf arteries and veins. Examinations were performed by 3 registered vascular technologists.

VTE mechanical prophylaxis was administered. Since the morning of KT surgery, patients had worn a graduated compression stocking with an ankle pressure of 20-30 $\mathrm{mmHg}$ for 1 month. In addition, patients were equipped with 3-segment (ankle, calf, and thigh) pneumatic compression device immediately after surgery for 2 weeks. Other pharmacologic prophylaxis was not administered.

\section{Immunosuppression therapy}

Either basiliximab (20 $\mathrm{mg}$ on operative day and postoperative day 4) or ATG (1.25 mg/kg for 5 days) was used as induction agent. Tacrolimus (trough level, $7-10 \mathrm{mg} / \mathrm{mL}$ ), mycophenolate mofetil ( $1.5 \mathrm{~g} /$ day), and prednisolone $(30 \mathrm{mg} / \mathrm{day}$, gradually tapered) were used for maintenance. For patients who underwent $\mathrm{ABO}$-incompatible KT and HLA-incompatible KT, thus requiring desensitization treatment prior to KT, additional treatment with rituximab and plasmapheresis following lowdose intravenous immunoglobulin therapy $(100 \mathrm{mg} / \mathrm{kg})$ was provided prior to KT. To prevent opportunistic infection, fluconazole $(50 \mathrm{mg} / \mathrm{day})$ and trimethoprim/sulfamethoxazole ( $800 \mathrm{mg} /$ day) were administered for 3 months.

\section{RESULTS}

\section{Baseline characteristics}

A total of $862 \mathrm{KT}$ cases (living donor KT, 551; deceased donor KT, 311) were performed between February 2010 and April 2016, and 829 recipients were analyzed in this study (Fig. 1). DVT occurred in 60 out of 829 cases (7.2\%). In detail, there were 11 cases (18.3\%) of proximal vein thrombosis and 49 cases (81.6\%) of IDDVT. All IDDVT cases were asymptomatic. The majority of diagnoses were established within 2 weeks (26 cases, 53\%), and there were 9 cases with delayed onset (diagnosed at 3-6 months after KT) (Fig. 3).

Either anticoagulation-first strategy or surveillancefirst strategy was employed in 49 IDDVT patients, based on diagnosis time and thrombus burden (Fig. 4). A total of 
19 patients were diagnosed with IDDVT during period 1 (February 2010-June 2013). (A) Of these patients, 5 exhibited low thrombus burden $(\leq 3 \mathrm{~cm}$ in length) in one calf vein and were therefore treated using surveillance-first strategy without anticoagulation. (a) A total of 30 patients were diagnosed with IDDVT during period 2 (July 2013-April 2016). (B) One of these patients exhibited $\geq 3$ relatively larger thrombi $(\geq 5 \mathrm{~cm}$ in length) and was thus treated using anticoagulation-first strategy. (b) In brief, 15 IDDVT patients $(A-a+b)$ and 34 patients $(B-b+a)$ were treated using anticoagulation-first and surveillance-first

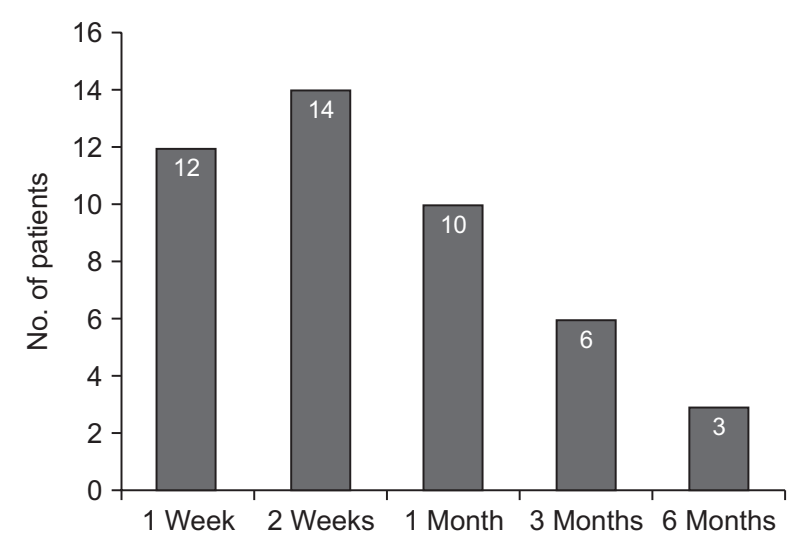

Fig. 3. Patient distribution according to a time of isolated distal deep vein thrombosis (IDDVT) diagnosis. Most of IDDVT diagnosed within 1 month after kidney transplantation. However, delayed type of IDDVTs were occurred till 6 months later. strategies, respectively.

The general characteristics of all IDDVT patients were assessed (Table 1). The male-to-female ratio was roughly $50: 50$, and the mean age was 54 years, with 13 patients aged $\geq 60$ years. Approximately $60 \%$ of patients received regular

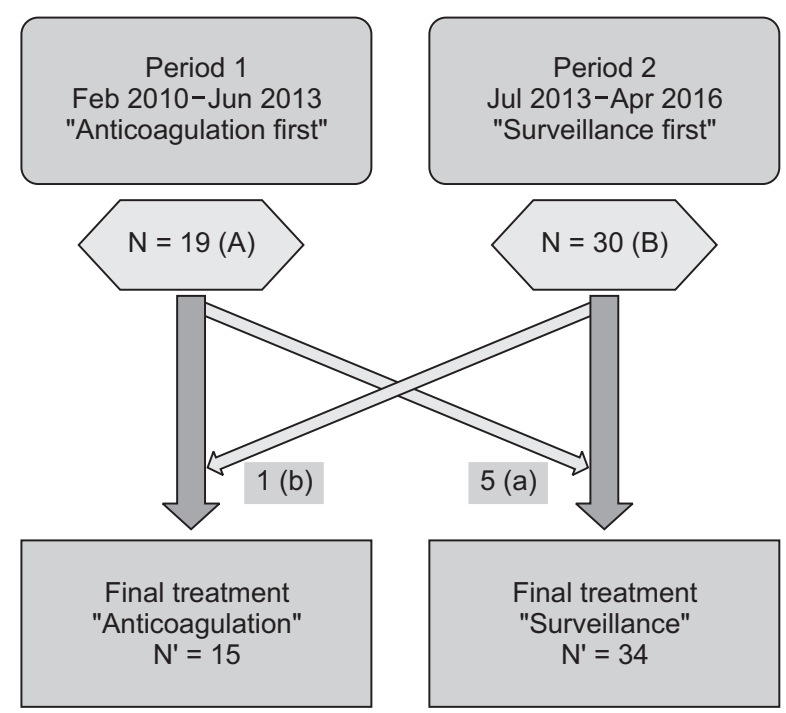

Fig. 4. Schematic descript of patient allocation. Nineteen patients were diagnosed with isolated distal deep vein thrombosis (IDDVT) during period 1 and 30 patients were in period 2. (A and B) According to the specific indications (a and b), overall 15 patients were treated by anticoagulationfirst strategy and 34 patients were managed by surveillance first strategy.

Table 1. Baseline characteristics of 49 IDDVT patients

\begin{tabular}{lccc}
\hline \multicolumn{1}{c}{ Characteristic } & Total $(\mathrm{N}=49)$ & Anticoagulation-first strategy $(\mathrm{n}=15)$ & Surveillance-first strategy $(\mathrm{n}=34)$ \\
\hline Male sex & $26(53)$ & $9(60)$ & $17(50)$ \\
Age $(\mathrm{yr})$ & $54 \pm 8.7(31-72)$ & $52.9 \pm 6.5(41-66)$ & $54.5 \pm 9.5(31-72)$ \\
BMI $\left(\mathrm{kg} / \mathrm{m}^{2}\right)$ & $22.8 \pm 3.3(15.5-34.8)$ & $23.5 \pm 3.8(18.7-34.8)$ & $22.4 \pm 3.0(15.5-29.8)$ \\
Cause of ESRD & & & $8(23.5)$ \\
DM & $11(22.4)$ & $3(20)$ & $10(29.4)$ \\
Hypertension & $14(28.6)$ & $4(26.7)$ & $9(26.5)$ \\
CGN & $14(28.6)$ & $5(33.3)$ & $7(20.6)$ \\
Others/unknown & $10(20.4)$ & $3(20)$ & $0(0)$ \\
History of VTE & $1(2.0)$ & $1(6.7)$ & $3(8.8)$ \\
History of malignancy & $4(8.2)$ & $1(6.7)$ & $1(2.9)$ \\
Hormonal therapy & $2(4.1)$ & $1(6.7)$ & $18(52.9)$ \\
Erythropoietin & $29(59.2)$ & $11(73.3)$ & $26(76.5)$ \\
Renal replacement therapy & & & $3(8.8)$ \\
Hemodialysis & $34(69.4)$ & $8(53.3)$ & $5(14.7)$ \\
Peritoneal dialysis & $9(18.4)$ & $1(6.7)$ & $10(29.4)$ \\
Preemptive & $6(12.2)$ & $7(46.7)$ & $67.2 \pm 64.6(0.8-251.1)$ \\
Duration of dialysis (mo) & $64.5 \pm 61.7(1-251.2)$ & $58.9 \pm 54.4(1.6-176.8)$ & $10(29)$ \\
Antiplatelet agent & $17(34.5)$ & &
\end{tabular}

Values are presented as number (\%) or mean \pm standard deviation (range).

IDDVT, Isolated distal deep vein thrombosis; BMI, body mass index; ESRD, end stage renal disease; DM, diabetes mellitus; CGN, chronic glomerulonephritis; VTE, venous thromboembolism. 
erythropoietin injection from dialysis, and hemodialysis was the major modality of renal replacement therapy. In addition, 34\% of patients used an antiplatelet agent to manage underlying vascular diseases. Only $2 \%$ of patients had a history of VTE, whereas $10 \%$ of patients had a history of malignancy. Comparison between the anticoagulation-first strategy group and the surveillance-first strategy group indicated no remarkable differences between both groups, except for the following: higher frequency of erythropoietin and antiplatelet agent use in the anticoagulation-first strategy group and greater proportion of patients with longer duration of preoperative hemodialysis in the surveillance-first strategy group.

With respect to transplant-related data, approximately $25 \%$ of patients had a history of transplantation, and $12 \%$ of patients underwent immunologically high-risk KT (either HLAincompatible or ABO-incompatible) (Table 2). The numbers of living and deceased donor transplants were similar, and $16 \%$ of patients exhibited delayed graft function. Both the

Table 2. Transplant-related data

\begin{tabular}{|c|c|c|c|}
\hline Variable & Total $(\mathrm{N}=49)$ & Anticoagulation-first strategy $(\mathrm{n}=15)$ & Surveillance-first strategy $(\mathrm{n}=34)$ \\
\hline Prior transplant history & $12(24.5)$ & $2(13.3)$ & $10(29.4)$ \\
\hline Living donor $\mathrm{KT}$ & $27(55.1)$ & $8(53.3)$ & $19(55.9)$ \\
\hline HLA mismatch number & $3.1 \pm 1.8(0-6)$ & $3 \pm 1.9(0-6)$ & $3.1 \pm 1.8$ \\
\hline Immunologically high-risk KT & $6(12.2)$ & $1(6.7)$ & $5(14.7)$ \\
\hline \multicolumn{4}{|l|}{ Induction immunosuppression } \\
\hline ATG & $11(22.4)$ & $1(6.7)$ & $10(29.4)$ \\
\hline Basiliximab & $38(77.6)$ & $14(93.3)$ & $24(70.6)$ \\
\hline \multicolumn{4}{|l|}{ Postoperative graft function } \\
\hline Standard & $41(83.7)$ & $13(86.7)$ & $28(82.4)$ \\
\hline Delayed & $8(16.3)$ & $2(13.3)$ & $6(17.6)$ \\
\hline 2-Year patient survival & $49(100)$ & $15(100)$ & $34(100)$ \\
\hline 2-Year graft survival & $49(100)$ & $15(100)$ & $34(100)$ \\
\hline
\end{tabular}

Values are presented as number (\%) or mean \pm standard deviation (range).

KT, kidney transplantation; HLA, human leukocyte antigen; ATG, antithymocyte globulin.

Table 3. Laboratory finding for evaluating coagulation status

\begin{tabular}{|c|c|c|c|c|c|}
\hline \multirow{2}{*}{ Variable } & \multicolumn{2}{|r|}{ Non-VTE KTR } & \multicolumn{2}{|r|}{ IDDVT KTR } & \multirow{2}{*}{ P-value } \\
\hline & No. & Value & No. & Value & \\
\hline \multicolumn{6}{|l|}{ Preoperative } \\
\hline Hematocrit (\%) & 579 & $31 \pm 5.3(16.8-52.3)$ & 49 & $32 \pm 5.3(19.9-46.7)$ & 0.239 \\
\hline Platelet $\left(/ \mathrm{mm}^{3}\right)$ & 579 & $182.8 \pm 61.2(53-423)$ & 49 & $180 \pm 63.5(71-314)$ & 0.824 \\
\hline PT (INR) & 579 & $1.05 \pm 0.16(0.87-4.4)$ & 49 & $1.04 \pm 0.09(0.91-1.44)$ & 0.416 \\
\hline Protein C deficiency & 571 & $32(5.8)$ & 33 & $1(3)$ & 0.715 \\
\hline Protein S deficiency & 571 & $134(23.4)$ & 33 & $7(21.2)$ & 0.242 \\
\hline AT III deficiency & 575 & $183(31.8)$ & 32 & $9(28.1)$ & 0.335 \\
\hline Factor V Leiden mutation & 572 & $0(0)$ & 31 & $0(0)$ & \\
\hline Prothrombin 20210A mutation & 558 & $0(0)$ & 31 & $0(0)$ & \\
\hline Lupus anticoagulant & 571 & $8(1.4)$ & 32 & $2(6.3)$ & 0.095 \\
\hline Anticardiolipin antibody & 573 & $20(3.4)$ & 32 & $0(0)$ & 0.617 \\
\hline Homocysteinemia & 570 & $389(68.2)$ & 32 & $18(56.3)$ & 0.56 \\
\hline D-dimer $(\mu \mathrm{g} / \mathrm{mL})$ & 565 & $1.22 \pm 1.23(0.07-14.4)$ & 32 & $1.48 \pm 1.04(0.19-4.3)$ & 0.072 \\
\hline \multicolumn{6}{|l|}{ Postoperative } \\
\hline D-dimer, 1 wk & 567 & $2.4 \pm 1.93(0.1-20.09)$ & 47 & $3.05 \pm 1.52(0.39-6.89)$ & $<0.001$ \\
\hline D-dimer, 2 wk & 570 & $1.96 \pm 1.94(0.17-25.56)$ & 34 & $3.0 \pm 2.16(0.26-12.14)$ & $<0.001$ \\
\hline D-dimer, 1 mo & 541 & $1.8 \pm 2.22(0.08-35.2)$ & 35 & $2.3 \pm 1.84(0.14-9.41)$ & 0.031 \\
\hline
\end{tabular}

Values are presented as mean \pm standard deviation (range) or number (\%).

VTE, venous thromboembolism; KTR, kidney transplant recipient; IDDVT, Isolated distal deep vein thrombosis; PT (INR), prothrombin time (international normalized ratio); AT III deficiency, antithrombin III deficiency. 
2-year graft survival rate and patient survival rate were $100 \%$. Intergroup comparison showed that there were more patients in the surveillance-first strategy group who had a history of organ transplantation, were immunologically at high risk, and received ATG induction therapy.

Blood coagulation test was performed in most cases until December 2014 but had not been conducted since then owing to health insurance coverage issues. Therefore, the comparison of outcomes in this study only included the data of patients who underwent blood test. We compared the results of IDDVT KTR to Non-VTE KTR (Table 3). Commonly observed abnormalities associated with coagulation factors were hyperhomocysteinemia, antithrombin III deficiency, and protein S deficiency in not only IDDVT KTR but also in Non-VTE KTR. The level of D-dimer was increased in both groups before- and after transplant surgery, and it was much remarkable in IDDVT KTR after transplant surgery with statistical significance.

\section{Outcome of IDDVT management}

Patients belonging to the anticoagulation-first strategy group were first treated with low-molecular-weight heparin (enoxaparin, $1 \mathrm{mg} / \mathrm{kg} \mathrm{q} 12$ hours), which was subsequently changed to warfarin, rivaroxaban, or apixaban for maintenance. Anticoagulant therapy was maintained for $\geq 3$ months in 11 patients $(73.3 \%$ ) but was discontinued in the remaining 4 patients owing to major bleeding or preparation for invasive procedures. Major bleeding was occurred in 3 patients; one had melena and 2 experienced hematuria and all of symptoms non-fatal and did not need transfusion. One patient was experienced recurrent urinary tract infection and needed multiple times of urinary tract interventions so we stopped anticoagulation and could not restart it. No disease progression was observed in any of the 15 patients.

In the surveillance-first strategy group, 4 out of 34 patients (11.7\%) exhibited disease progression on follow-up DUS. Although none of the 4 patients exhibited extension into the proximal vein, all of them showed increased thrombus range within the calf vein or increased number of calf veins with thrombus. These patients received anticoagulant therapy immediately after disease progression was observed: 2 of the patients received anticoagulant therapy for $\geq 3$ months, whereas the other 2 patients stopped treatment at 2 weeks (patient needed graft biopsy 2 times and multiple sessions of plasma exchange for rejection treatment) and 1 month (noncompliance).

Overall, 13 out of 49 IDDVT patients (26.5\%) received standardized anticoagulant therapy for $\geq 3$ months, whereas the remaining 36 IDDVT patients (73.5\%) either received insufficient anticoagulant therapy (6 patients, $16.6 \%$ ) or did not receive anticoagulant therapy at all (30 patients, $83.3 \%$ ). Followup DUS was performed at 1 year after KT, and progression into the proximal vein was not observed throughout the follow- up period. At a mean follow-up period of 55 months (26-96 months), no case of symptomatic VTE was detected.

\section{DISCUSSION}

According to a nationwide database, the incidence rate of VTE in the Western general population has been reported to be $0.1 \%-0.2 \%$. In cases of VTE occurrence, anticoagulant therapy must be provided for 3 months to prevent the onset of acute pulmonary thromboembolism and consequent mortality, as well as the extension and recurrence of VTE $[6,14]$. A lack of appropriate treatment has been shown to increase the mortality by $\sim 50 \%$ and extension or recurrence by $\sim 63 \%$ [14]. Compared with the general population, KTR reportedly exhibits an increased incidence rate of VTE $(6.2 \%-8.3 \%)$ owing to multifactorial effects $[9,12,13,15]$. As KTR is more susceptible to VTE and the occurrence of VTE can be an important risk factor affecting both patient and graft survival, suitable treatment is essential. However, limited choices as to anticoagulant therapy are available because of multiple reasons, including the patient's postoperative state, interaction with other medications, and possible requirement for frequent invasive procedures in the future. For proximal DVT, there is no need for debate, as anticoagulation is an established standard treatment protocol. However, for IDDVT, in which a decision about anticoagulation and surveillance strategies needs to be made, arriving at a decision on whether the risk of VTE or anticoagulation should be taken is difficult. In our study, we described the experiences from these 2 strategies in detail, which we believe will assist physicians who will encounter the same issue. Our strategy for managing IDDVT has been changed as time passed. We recognized the clinical importance of IDDVT, but could not assure how to treat at first. In early stage, we preferred to use anticoagulants as soon as a patient was diagnosed IDDVT. During that time, thrombus progression was not developed at all and we changed our strategy from anticoagulation-first to surveillance-first at 2013.

In this study, $30 \%$ of patients with post-KT IDDVT received anticoagulant therapy, and neither disease progression nor recurrence was observed. The remaining $\sim 70 \%$ of patients either received insufficient anticoagulant therapy ( $<3$ months) or did not receive anticoagulant therapy at all; nevertheless, none of them exhibited progression into the proximal vein or recurrence. Therefore, we believe that pharmacologic prophylaxis is not mandatory for managing IDDVT, surveillance and appropriate maintenance of mechanical prophylaxis are appropriate treatment protocol for IDDVT in KTR. The findings of our study suggest that it is not too late to provide anticoagulant therapy for patients with confirmed disease progression under follow-up.

Nonetheless, precautions should be taken for interpreting 
the outcomes of this study. In this study, the study cohort was limited to Koreans, who exhibit a lower incidence rate of VTE and are less vulnerable than Caucasians or Africans. Several studies have previously reported a racial difference in the incidence rate of VTE [16-20]. The incidence rate of VTE in our study was $7.2 \%$, which seems similar to that in Western KTR. However, $<20 \%$ of patients with VTE had proximal vein involvement, whereas the remaining $80 \%$ had asymptomatic IDDVT. Most studies assessing the incidence of VTE only included subjects with symptoms and consequent diagnosis of the condition; in comparison, our study performed screening DUS for the entire patient cohort and included cases of asymptomatic VTE. Thus, the incidence rate was as high as that in the Western population. If screening was performed in the Western population, the incidence rate of IDDVT will likely be much higher than that in our study.

In addition, considering that the patients analyzed in our study had asymptomatic IDDVT diagnosed via screening DUS, the treatment outcome and prognosis may be different from those in patients diagnosed with symptomatic IDDVT in real clinical setting. Therefore, patients with symptomatic IDDVT have higher probability of having extensive lesions than the patients in our study. Hence, it is difficult to generalize our study findings to all IDDVT patients. There are well-known symptoms of IDDVT, and the provision of anticoagulation-first treatment may be beneficial for patients with high thrombus burden in order to prevent proximal propagation and reduce future recurrence $[4,5]$. Furthermore, anticoagulant therapy should be regarded as the priority treatment if patients do not prefer the use of compression device or ambulation or if surveillance is not a readily available option.

In this study, we analyzed the laboratory findings associated with coagulation status of KTR. In KTR, anemia, hyperhomocysteinemia, protein $S$ deficiency, and antithrombin III deficiency were common. The level of D-dimer was elevated and it had 2 traits. First, the mean level of D-dimer was higher than normal $(<0.5 \mu \mathrm{g} / \mathrm{mL})$ even before transplant surgery and only 154 (25.7\%) were within normal range among total 597 KTR (Table 3). In general, D-dimer increases in nonspecific, various situations so it is not useful to diagnose VTE but can be meaningful to rule it out. In renal impairment patients, however, D-dimer has limited value to exclude a possibility of VTE. Second, the level of D-dimer was much increased in IDDVT KTR after transplant surgery compared to Non-VTE KTR with statistical significance. However, it has been well known that a level of D-dimer was not correlated with disease course or severity so we didn't analyze it further such as obtaining cutoff level.

In presented results, factor $\mathrm{V}$ Leiden mutation or prothrombin 20210A gene mutation was not detected. These gene mutation has never been reported in Korea. The Korean
Society for Vascular Surgery released practice guideline in 2016 and recommended that not to do these gene test in Korean [21].

Lastly, there exist few limitations on the treatment method and outcomes owing to our retrospective study design. First, differences in baseline patient characteristics were analyzed based on the fact that this study is a historical comparisonbased study. In addition, there were more patients during period 2 who had a history of organ transplantation, were immunologically at high risk, and received ATG induction therapy. We believe that the differences between the 2 periods were attributable to the accumulated organ transplantation experience during period 2, compared with that during period 1. Consequently, the number of immunologically high-risk KT and deceased donor KT from marginal donors increased during period 2; therefore, the number of ATG induction therapy also increased. Furthermore, the laboratory test for the evaluation of coagulation status was insufficient owing to the retrospective nature of this study, preventing it from keeping the number of patients consistent.

In conclusion, this study suggested that serial DUS surveillance along with the maintenance of mechanical prophylaxis could be effective methods for preventing thrombus proximal extension and recurrence in asymptomatic IDDVT KTR. However, patients with symptomatic lesions or multiple, high-burden lesions may require anticoagulation. Close inspection with high suspicion and serial assessment to confirm thrombus characteristics will support a decision making.

\section{ACKNOWLEDGEMENTS}

\section{Conflict of Interest}

No potential conflict of interest relevant to this article was reported.

\section{ORCID iD}

Mi-Hyeong Kim: https://orcid.org/0000-0002-6190-085X

Kang-Woong Jun: https://orcid.org/0000-0002-0055-1115

Jeong-Kye Hwang: https://orcid.org/0000-0001-7146-6957

Sun-Cheol Park: https://orcid.org/0000-0003-2701-0180

Sang-Seop Yun: https://orcid.org/0000-0001-7960-0444

In-Sung Moon: https://orcid.org/0000-0002-5543-6755

Ji-Il Kim: https://orcid.org/0000-0001-5910-6916

\section{Author Contribution}

Conceptualization: SSY, ISM, JIK

Formal Analysis: JKH, SCP

Investigation: MHK, KWJ

Methodology: MHK, JKH

Project Administration: MHK, KWJ

Writing - Original Draft: MHK, KWJ, JKH 
Writing - Review \& Editing: SCP, SSY, ISM, JIK

\section{REFERENCES}

1. Solis MM, Ranval TJ, Nix ML, Eidt JF, Nelson CL, Ferris EJ, et al. Is anticoagulation indicated for asymptomatic postoperative calf vein thrombosis? J Vasc Surg 1992;16:414-8.

2. Macdonald PS, Kahn SR, Miller N, Obrand D. Short-term natural history of isolated gastrocnemius and soleal vein thrombosis. J Vasc Surg 2003;37:523-7.

3. Schwarz T, Buschmann L, Beyer J, Halbritter K, Rastan A, Schellong S. Therapy of isolated calf muscle vein thrombosis: a randomized, controlled study. J Vasc Surg 2010;52:1246-50.

4. Deitcher SR, Caprini JA. Calf deep venous thrombosis should be treated with anticoagulation. Med Clin North Am 2003;87:1157-64.

5. Masuda EM, Kistner RL. The case for managing calf vein thrombi with duplex surveillance and selective anticoagulation. Dis Mon 2010;56:601-13.

6. Kearon C, Akl EA, Ornelas J, Blaivas A, Jimenez D, Bounameaux $\mathrm{H}$, et al. Antithrombotic therapy for VTE disease: CHEST guideline and expert panel report. Chest 2016;149:315-52.

7. Ahn S, Kim MH, Jun KW, Hwang JK, Park SC, Moon IS, et al. The incidence and risk factors for deep vein thrombosis after kidney transplantation in Korea: single-center experience. Clin Transplant 2015:29:1181-6.

8. Andrassy J, Zeier M, Andrassy K. Do we need screening for thrombophilia prior to kidney transplantation? Nephrol Dial Transplant 2004;19 Suppl 4:iv64-8.

9. Kazory A, Ducloux D. Acquired hypercoagulable state in renal transplant recipients. Thromb Haemost 2004:91:646-54.

10. Todeschini P, La Manna G, Dalmastri V, Feliciangeli G, Cuna V, Montanari M, et al. Incidence of late deep venous thrombosis among renal transplant patients. Transplant Proc 2013:45:2666-8.

11. Verhave JC, Tagalakis V, Suissa S, Madore F, Hebert MJ, Cardinal H. The risk of thromboembolic events in kidney transplant patients. Kidney Int 2014:85: 1454-60.

12. Wattanakit K, Cushman M, StehmanBreen C, Heckbert SR, Folsom AR. Chronic kidney disease increases risk for venous thromboembolism. J Am Soc Nephrol 2008;19:135-40.

13. Humar A, Johnson EM, Gillingham KJ, Sutherland DE, Payne WD, Dunn DL, et al. Venous thromboembolic complications after kidney and kidney-pancreas transplantation: a multivariate analysis. Transplantation 1998;65:229-34.

14. Kearon C, Akl EA, Comerota AJ, Prandoni P, Bounameaux H, Goldhaber SZ, et al. Antithrombotic therapy for VTE disease: antithrombotic therapy and prevention of thrombosis, 9th ed: American College of Chest Physicians evidence-based clinical practice guidelines. Chest 2012;141:e419S-
e496S.

15. Allen RD, Michie CA, Murie JA, Morris PJ. Deep venous thrombosis after renal transplantation. Surg Gynecol Obstet 1987;164:137-42.

16. White RH, Keenan CR. Effects of race and ethnicity on the incidence of venous thromboembolism. Thromb Res 2009;123 Suppl 4:S11-7.

17. Heit JA. The epidemiology of venous thromboembolism in the community: implications for prevention and management. J Thromb Thrombolysis 2006;21: 23-9.

18. Naess IA, Christiansen SC, Romundstad P, Cannegieter SC, Rosendaal FR, Hammerstrom J. Incidence and mortality of venous thrombosis: a population-based study. J Thromb Haemost 2007:5:692-9.

19. Lee LH, Gallus A, Jindal R, Wang C, Wu $C C$. Incidence of venous thromboembolism in Asian populations: a systematic review. Thromb Haemost 2017;117:224360.

20. Jang MJ, Bang SM, Oh D. Incidence of venous thromboembolism in Korea: from the Health Insurance Review and Assessment Service database. J Thromb Haemost 2011;9:85-91.

21. Min SK, Kim YH, Joh JH, Kang JM, Park UJ, Kim HK, et al. Diagnosis and treatment of lower extremity deep vein thrombosis: Korean practice guidelines. Vasc Specialist Int 2016;32:77-104. 